\title{
Diagnóstico de la redacción académica en estudiantes de la carrera de educación inicial de la universidad estatal península de Santa Elena
}

\author{
Greys Dayana Albán Zamora \\ greys.albanzamora@upse.edu.ec \\ Genesis Nicole Balón Cruz \\ genesis.baloncruz@upse.edu.ec \\ María José Campos Sarango \\ maria.campossarango@upse.edu.ec \\ Lady Mayte Clemente Clemente \\ lady.clementeclemente@upse.edu.ec \\ Ingrid Soledad Choez Beltran \\ ingrid.choezbeltran@upse.edu.ec \\ Evelyn Lisbeth Santistevan Merejildo \\ evelyn.santistevanmerejildo@upse.edu.ec \\ Carla Solange Santos Sandoval \\ carla.santossandoval@upse.edu.ec \\ Universidad Estatal Península de Santa Elena \\ Santa Elena - Ecuador
}

\section{RESUMEN}

El presente Artículo se planteó como objetivo: Diagnosticar la redacción académica en los estudiantes de la Carrera de Educación Inicial de la Universidad Estatal Península de Santa Elena - Ecuador. El estudio se encuentra enmarcado hacia un enfoque cuantitativo, investigación de campo con un alcance descriptivo trasversal. Para la recolección de datos se diseñó una encuesta con su respectiva escala de Likert, misma, que se aplicó a 81 estudiantes que cursan el Primer semestre de la Carrera de Educación Inicial. La confiabilidad del instrumento se la obtuvo, mediante, el Alfa de Cronbach con un valor de 0,903 que lo determina como aplicable. Los principales resultados determinan que el 84,6\% de estudiantes presentan dificultades de Ortografía, es decir, no emplean correctamente las reglas de la escritura durante el desarrollo de la redacción académica.

Palabras clave: redacción académica; gramática; ortografía; normas APA 


\title{
Diagnosis of academic writing in students of the early childhood education program at the Santa Elena Peninsula state university
}

\begin{abstract}
The objective of this article was to diagnose the academic writing skills of the students of the Early Childhood Education program at the Peninsula de Santa Elena State University - Ecuador. The study is framed towards a quantitative approach, field research with a transversal descriptive scope. For data collection, a survey was designed with its respective Likert scale, which was applied to 81 students in the first semester of the Early Childhood Education Career. The reliability of the instrument was obtained by means of Cronbach's Alpha with a value of 0.903 , which determines its applicability. The main results show that $84.6 \%$ of the students present spelling difficulties, that is, they do not correctly use the rules of writing during the development of academic writing.
\end{abstract}

Keywords: academic writing; grammar; spelling; APA standards

Artículo recibido: 20 diciembre. 2021 Aceptado para publicación: 10 enero 2022 Correspondencia: greys.albanzamora@upse.edu.ec Conflictos de Interés: Ninguna que declarar 


\section{INTRODUCCIÓN}

Los escritos académicos elaborados por estudiantes universitarios tienen como propósito fundamental expresar un conjunto de ideas de forma clara, precisa y elegante que conducen al lector a la comprensión de la profundidad, contexto e intención de lo que se desea comunicar. Por su parte, la redacción académica precisa de habilidades y destrezas que se logran en la práctica continua a través de un proceso crítico- reflexivo que requiere de tiempo y de elementos cognitivos en los que subyacen dominios teóricos para la articulación apropiada de ideas que sirven a la construcción de conceptos.

Es evidente, que la buena redacción se sustenta sobre la base de conocimientos amplios de las normas que rigen la escritura que se alcanzan durante el proceso educativo de la básica y bachillerato y que prepara al estudiante hacia los nuevos desafíos de la formación universitaria, sin embargo, cuando el docente no enfatiza en la importancia de la redacción académica para la formación profesional durante los procesos pedagógicos difícilmente el estudiante desarrollará las destrezas y habilidades de la escritura que le capacita para aportar con trabajos de calidad.

En este escenario, las dificultades en la redacción académica que presentan los estudiantes del primer semestre de la Carrera de Educación Inicial de la Universidad Estatal Península de Santa Elena es una situación que genera preocupación en los docentes porque afecta directamente la consecución de los resultados de aprendizaje de las asignaturas contempladas en la malla curricular; consecuentemente, al presentarse como una debilidad repercute en el rendimiento académico de los estudiantes.

Es claro, que la pandemia marcó un precedente importante en relación a la forma en cómo se estaban desarrollando los procesos educativos durante el año 2020, puesto que, la educación en Ambientes Virtuales de Aprendizaje (AVA) no satisface en su totalidad las necesidades de aprendizaje de los estudiantes, ya que, no todos cuentan con los recursos tecnológicos para conectarse regularmente a las clases y, por otro lado, la nueva modalidad de estudio implica procesos de autorregulación del aprendizaje más exigentes que todavía no se han alcanzado. Consecuentemente, la sobrecarga laboral en los docentes durante las jornadas virtuales y el poco manejo de herramientas tecnológicas ha impedido que desarrollen un seguimiento de calidad. En este escenario, es claro que, las habilidades de redacción académica en los estudiantes se han visto afectadas y que se demuestra en 
la forma en como articulan sus ideas, desarrollan conceptos y redactan sus trabajos académicos.

La redacción académica se la denomina como "un proceso de construcción de productos escritos, su aprendizaje y su práctica demandan un cuidadoso proceso de elaboración de su materia prima -el pensamiento-y de su forma de expresión o presentación por medio de textos escritos" (Basulto, 1998, como se citó en Espinoza, 2017, p.81). Este proceso es necesario para la creación de nuevas ideas, y, sobre todo, saber plasmarlas de forma escrita, de manera que, pueda trasmitir sus conocimientos, además, de identificar sus errores para corregirlos de manera honesta y sincera.

En el contexto universitario, Espinoza Centellas (2017) comenta que la redacción académica tiene gran relevancia en los estudiantes ya que, escribir es algo que puede ser cotidiano y elemental, sin embargo, no es sencillo; en esta etapa, los trabajos son más formales y complejos, por los textos que deben escribir como son los resúmenes, trabajos de investigación, ensayos, foros, proyectos, artículos científicos, hasta tesis de grado. (pp. 81- 82)

Según Gutiérrez y Flórez (2011) en la redacción se “explica tres subprocesos en el acto de escribir: la planeación (pensar el texto, generar ideas, anticipar quién va a leerlo), transcripción/traducción (primera aproximación al texto definitivo, poner las ideas en el papel) y revisión/edición (perfeccionamiento sucesivo de la primera versión)" (pp. 140 141). La formación académica superior permite adquirir, reforzar y aplicar todos estos procesos de los cuales se deben tener muy en claro para que pueda llevarlo a cabo en su día a día.

El docente por lo general “evalúa la calidad de la redacción, el uso correcto de los signos de puntuación, párrafos coherentes, empleo del léxico y coherencia adecuada de acuerdo con el contexto, como la lógica de las ideas" (Ñañez y Lucas, 2017, p. 796). Por ende, la Institución educativa junto a los profesores asumen la responsabilidad de direccionar a sus estudiantes hacia aprendizajes profundos y, en proponer capacidades que impliquen procesos cognitivos que les permitan asumir situaciones desafiantes.

Por otra parte, los alumnos no solo aprenderán a escribir, sino más bien serán capaces de ir mejorando otras destrezas comunicativas cuando compartan e intercambien ideas, por lo general, se entiende que, aprender a escribir significa aprender a organizar y construir 
textos con coherencia lógica, adaptándolo de acuerdo con el destinatario, el tema tratado y el tipo de texto. (Palma, 2012, como se citó en Montesdeoca et al., 2021, pp. 1 - 21).

En los escritos la argumentación se vuelve "una herramienta fundamental para la producción de textos académicos y científicos" (Araque et al., 2019, p. 54). Esto permite una mejor comprensión e interpretación de ideas al momento de plasmarla de manera escrita, dando concordancia y lógica en los trabajos redactados.

Con base en lo que comenta Ñañez y Lucas (2017) para la producción textual es necesario tomar en cuenta los siguientes requerimientos: seleccionar un tema central, que permita construir ideas basándose en una propiedad léxica, para formar una unidad temática que se desglosará a medida que se redacte; seguido del uso adecuado de las normas ortográficas, gramaticales, mayúsculas y signos de puntuación, que generará coherencia y claridad del texto, obteniendo así una eficiente comunicación (pp. 800- 801).

Todo trabajo académico que se quiera realiza se inicia por la elección del tema, en algunas ocasiones el docente es quién da indicaciones del tema a abordar. A partir de esto, es importante, tener conocimiento de lo que se va a redactar, "cuando el estudiante se prepara es necesario considerar el asunto sobre el que va a escribir, esta preparación comprende todo el sistema de relaciones contextuales, socioculturales, e históricas alrededor del tema, así como su propia experiencia” (González et al., 2019, pp. 14 - 26). Tomando en consideración la idea de los autores es fundamental saber qué, al momento de redactar se debe determinar el contexto y qué elementos lingüísticos son relevantes en este caso.

Posteriormente se prosigue a la construcción de texto, este tema ha llegado a ser una de las dificultades comunicativas de los estudiantes en los diferentes niveles de enseñanza, por lo que requiere una minuciosa atención en su elaboración ya que es "un complejo proceso de organización y estructuración de las ideas y niveles del texto, para comunicar un mensaje por escrito, poniendo de manifiesto la competencia comunicativa del que escribe" (Castro y Pérez, 2018, p. 7 - 11). Una vez determinado el tema es necesario ordenar adecuadamente las palabras y frases, para así conformar la unidad de texto, la cual permitirá ordenar con exactitud las ideas.

Para poder llevar a cabo dicha construcción, es necesario la comprensión lectora por parte del estudiantado, puesto que, implica diversos procesos de decodificación de la información que se está leyendo, usualmente: 
El lector se enfrenta a varios elementos que le suministran información como: letras, palabras, grafías, desde un nivel básico, que le permite a la vez, avanzar al nivel superior por medio de acciones como: encontrar y darles significado a las palabras, sentido a las frases, captación del sentido general del texto y contexto que aborda la lectura. (Llamazares et al., 2013 como se citó en Mantilla y Barrera, 2021, pp. 142 - 163)

Por ende, la comprensión lectora va más allá de la acción de leer o interpretar textos, se trata de proceso de construcción de pensamientos empleando los conocimientos y vivencias previas del lector.

"Las estrategias metodológicas de la comprensión lectora se estructuran en tres fases que intervienen en su comprensión: prelectura (presentar, lectura superficial, predicción y activación de conocimiento previo), lectura (tomar notas y encontrar información) y pos-lectura (comprensión, estudio, propósito, etc.)". (Brown y Lee, 2005 como se citó en Montesdeoca et al., 2020, pp. 49 - 60).

Para Zorrilla (2005, como se citó a Sánchez y Silva, 2021) manifiesta cinco niveles fundamentales para la comprensión lectora: el primero es el nivel literal, que permitirá reconocer y recordar los detalles precisos del texto como personajes, nombres, lugar, entre otros; el segundo nivel es de reorganización de la información, se basa en la capacidad de extraer la información del texto y crear esquemas; el tercer nivel es de comprensión inferencial, que consiste en crear hipótesis o suposiciones de lo leído; el cuarto nivel se encuentra la lectura crítica, consiste en realizar juicio de valor sobre el texto, y a su vez, comparándolo con sus conocimientos; el último nivel es la apreciación lectora, que comprende en las cuestiones de estética u opinión respecto al texto. (p. 4)

Por otro lado, Cromley (2014 como se citó en González et al., 2016) expone que el vocabulario es elemental para el desempeño de la comprensión lectora, pues no conocer el uso o sentido de determinadas palabras se convierte en un obstáculo importante para el logro de una representación integrada que dé cuenta de la comprensión (pp. 1 - 18). Por esta razón conocer los significados de las palabras permite establecer conexiones sobre las ideas del texto y relacionarlas con su propio conocimiento previo.

En la redacción, "la expresión escrita constituye una actividad comunicativa que integra procesos mentales, lingüísticos, y estilísticos complejos, en cuya interrelación descansa 
la efectividad de la comunicación en el código escrito" (González et al., 2019, pp. 14 24). Este código integra un sistema de signos que permitirá transcribir el código oral, para ello se requiere una revisión ortográfica y gramática, la que posibilitará una comunicación entre los lectores por el uso de un código común.

Es así que las normas ortografícas y gramaticales, son consideradas componentes importantes para los trabajos académicos. Por lo que menciona Cabanillas Huam (2021) que, la ortografía se fundamenta pedagógicamente al formar parte de una competencia comunicativa de carácter universal, por ende, se resalta el uso correcto para lograr una comunicación oral apropiada, además, la producción y comprensión de los textos es transcendental para el éxito académico de los estudiantes y su desenvolvimiento en el entorno social. (pp. $457-475$ ).

En los escritos, es primordial que se apliquen este conjunto de reglas, como menciona Veliz Jara (2020) que dichas normas ortográficas establecen la forma en la que han de ser escritas las palabras, y es fundamental en el proceso de aprendizaje, ya que estandarizan la forma de escribir en una comunidad de hablantes y además, posibilita la comprensión, por parte de otros. Por lo que se puede destacar que la ortografía consiste en:

Un sistema alfabético, sistema de grafemas, habitualmente llamados letras, que tienen una gran variedad de formas y estilos. También incluye las reglas ortográficas y de puntuación, mediante las cuales, las letras pueden cambiarse para representar los sistemas fonéticos, morfofonémicos, morfémicos, sintácticos y pragmáticos del lenguaje. La ortografía debe indicar a través de la puntuación las pautas de oración, frase y cláusula que representan significado. (Ferreiro, 1986, como se citó en Montesdeoca et al., 2021)

Por lo mencionado se debe considerar que la ortografía influye en la redacción, una palabra mal escrita, errores de acentuación y puntuación pueden generar que la idea del texto sea mal interpretada o no tenga sentido. Debido a esta dificultad Benítez Almirón (2015) manifiesta que es ncesario que el estudiante incluya en sus actividades de escritura, estrategias como leer, analizar, escribir, examinar, corregir, reescribir el texto cuantas veces sea necesario, etc., para así lograr el dominio de sus habilidades escriturales (p.30). De este modo las ideas que se plantean en los textos se presentarán de manera clara, pertienente y congruente proporcionando así una buena comprensión e interpretación. 
En la expresión escrita se debe considerar los procesos de escritura, así mismo, investigar y seleccionar fuentes de información confiables y relevantes, para que más adelante, facilite la organización de las ideas.

La presentación de los trabajos, como menciona (Durán, et al., 2017) que debe seguir criterios de acuerdo con la normativa académica de la institución, como el plazo de presentación, estructura, normas de estilo, entre otras. La misma que debe tener calidad científica y técnica, debe resaltar la originalidad, es decir, en ningún caso puede haber sido copiado total o parcialmente, sino que debe constituir una obra creativa e inédita del estudiante (p. 12). En todo documento de carácter académico, el estudiante debe aprender a seleccionar varias fuentes de información e investigación, para poder comprender, deducir, analizar, sintetizar.

De acuerdo con Gallego Valarezo (2017) las normas APA cumplen la función de andamiaje en la elaboración de trabajos de investigación estos pueden ser: artículos científicos, ensayos, tesis de grado, monografías. Durante la realización del trabajo las normas APA son una guía que permite la adaptación y correcta aplicación del formato para elaborar trabajos académicos (pp. 17 -19).

Por lo general, el estilo APA se emplea con frecuencia en los trabajos académicos, el mismo que, a su vez, dispone de ciertos requerimientos como el estilo, citación, referencia bibliográfica, tablas, gráficos, etc. Las Normas APA (American Psychological Association) son un conjunto de directrices diseñadas para facilitar una comunicación clara y precisa en las publicaciones académicas. "Las normas APA son una guía que sirve para seguir y respetar las reglas de un escrito y, así mismo, no recurrir en plagio y dar credibilidad al autor permitiendo conocer de donde se obtiene la información" (Moreno y Carrillo, 2019, p. 26).

La presente investigación se planteó como objetivo: Diagnosticar el nivel de redacción académica en los estudiantes de primer semestre de la Carrera de Educación Inicial de la Universidad Estatal Península de Santa Elena.

\section{ESTRATEGIAS METODOLÓGICAS O MATERIALES Y MÉTODOS}

La investigación esta direccionada hacia un enfoque de investigación cuantitativa, que "se asocia con la medición, o sea, con el acto de asignar números de acuerdo con reglas, objetos, sucesos o fenómenos" (Cerda Gutierrez, 1993, p. 46). 
Con base a esta definición, se infiere que la investigación cuantitativa tiene la capacidad de aumentar o disminuir los datos, ya que, está vinculada con la cantidad y la orientación de la comprobación estadística, a partir de, una magnitud, extensión o cantidad determinada, así mismo, busca establecer relaciones causales entre variables a través de la prueba de hipótesis.

Se utilizó la investigación de tipo descriptivo, con el fin de, detallar características o propiedades importantes del sujeto de estudio. Para Sampieri (2014), el diseño no experimental depende del tiempo en que se recolectan los datos, el alcance transversal, reúne todos los datos en un solo momento, su intención es describir las variables y analizar sus acontecimientos (pp. 154-155).

Junto con una investigación de campo, que para Monje (2011) explica que es un proceso por el que el investigador va accediendo progresivamente a la información fundamental para su estudio. (p.44)

Para el siguiente estudio, la población estuvo conformada por los universitarios de primer Semestre de la Carrera de Educación Inicial Facultad de Ciencias de la Educación e Idiomas en la Universidad Estatal Península de Santa Elena, La Libertad, en el periodo académico 2021 - 2. La muestra seleccionada fue de 81 estudiantes, cuyas edades oscilan entre los 17 y 28 años.

Para la recolección de datos se utilizó la encuesta que se define como una técnica que engloba un conjunto de procedimientos estandarizados, a través, de los cuales se recaudan y analizan los datos obtenidos de una muestra de la población para analizar y reconocer características. La misma que se realizó de manera virtual.

La encuesta consta de 23 ítems con su respectiva escala de Likert con los siguientes valores: nunca (1), raramente (2), ocasionalmente (3), frecuentemente (4), muy frecuentemente (5). Por otra parte, se consideró los siguientes ejes temáticos que son los principales requerimientos que se debe tener presente en todo trabajo de redacción académica: La Ortografía, constituida por 8 preguntas; Comprensión lectora de 9 preguntas y Normas APA con 6 preguntas.

Los ejes mencionados, se muestran como relevantes durante el proceso de la redacción académica. La Comprensión Lectora es uno de los procesos esenciales que el estudiante debe dominar, para facilitar su conocimiento ante la lectura de un texto, de esta manera, se promueve el pensamiento crítico el cual permite al educando nutrir su aprendizaje. 
Hay que tener en cuenta que, para una adecuada redacción se muestra el dominio Ortográfico, considerando que una buena y correcta ortografía garantiza el éxito en el ámbito educativo y profesional, permitiendo así, expresar y plasmar ideas con un enfoque preciso, claro e incluso conciso, de modo que, el destinatario reciba el mensaje correcto, por lo que, se evitan confusiones en la comunicación y, por ende, cuidar la ortografía demuestra interés como, también, profesionalismo.

Así mismo, se consideró las normas Apa, ya que, tienen gran transcendencia en los trabajos académicos, porque permite analizar, citar, compartir y dar los méritos correspondientes a los autores durante la redacción académica; sin embargo, se tiende a generar diferentes situaciones que entorpecen la utilización de estas, al momento de aplicarlas en un texto académico.

El análisis de los datos obtenidos se lo realizó a través del software SPSS Statistics, mediante, una estadística descriptiva para hallar las medias de tendencia central (media, mediana, moda), frecuencias y desviación estándar. Adicionalmente, la confiabilidad de los análisis se lo realizó, mediante, el Alfa de Cronbach cuyo índice de fiabilidad arrojó un 0,903 , que determina como aplicable.

Para asegurar la validez del instrumento se realizó el juicio de experto. Según el criterio de (Galicia, et al., 2017, pp.42 - 45) la validez de contenido por juicio de expertos es una opinión informada de personas con trayectoria en el tema, que son examinadas por otros como expertos cualificados en éste, y que pueden dar información, evidencia, juicios y valoraciones.

\section{RESULTADOS Y DISCUSIÓN}

Se realizó la encuesta a 81 estudiantes universitarios de primer semestre de la Carrera de Educación Inicial de la Universidad Estatal Península de Santa Elena donde se obtuvieron los siguientes resultados de acuerdo a los ejes temáticos sobre redacción académica:

Tabla 1: Ortografía

\begin{tabular}{|c|c|c|c|c|c|c|}
\hline \multicolumn{3}{|c|}{ Semestre } & Frecuencia & Porcentaje & Porcentaje & Porcentaje \\
\hline \multirow{4}{*}{ Primero } & \multirow{4}{*}{ Válidos } & Débil & 20 & 24,7 & 24,7 & 24,7 \\
\hline & & Intermedio & 50 & 61,7 & 61,7 & 86,4 \\
\hline & & Alto & 11 & 13,6 & 13,6 & 100,0 \\
\hline & & Total & 81 & 100,0 & 100,0 & \\
\hline
\end{tabular}


De la tabla 1 se destaca los distintos porcentajes, respecto al eje temático de Ortografía en la que existe un porcentaje débil de $24,7 \%$ correspondiente a la falta de conocimiento ortográfico al realizar un escrito, los resultados de los educandos indican que presentan dificultades en la ortografía, esto se debe al "inadecuado manejo de las reglas ortográficas es un problema que afecta a muchos estudiantes de todos los niveles y se presenta con características específicas" (Cabanillas, 2021, p. 460). Debido a esto, no existe una comunicación clara y eficiente al momento de expresar sus ideas o redactar, es necesario conocer la importancia de la ortografía, pues, es la base para lograr interacción entre el escritor y lector.

El ítem intermedio es notorio su alto porcentaje representando en el 61, 7\%, que en ocasiones se toma en cuenta el uso correcto de la ortografía, como señala Gabarró (2011) es importante reflexionar sobre la relevancia de la ortografía en los procesos de enseñanza, puesto que, la misma va ligada con la escritura y el la correcta utilización de las palabras, de este modo, es posible la comunicación asertiva y la conceptualización de conceptos, dando paso a la correcta comprensión de textos académicos. Dichos estudiantes consideran la ortografía como una herramienta importante que favorece sus aprendizajes.

Mientras que el porcentaje alto corresponde solo a un 13,6\% del estudiantado con un nivel de ortografía excelente, debido a que en su proceso escolar han adquirido habilidades que les permiten comprender las diferentes normas y reglas que existen, según González (2012) una de las razones por las cuales los estudiantes sienten gran empatía por aprender ortografía es la forma en que el docente utiliza la metodología para enseñarla (pg. 186). Para abordar el conocimiento ortográfico es necesario que los docentes tengan una buena ortografía y gramática utilizando diferentes metodologías para trasmitirla y que tengan una precisión léxica para diferenciar el uso adecuado de las palabras en los distintos contextos.

Tabla 2 Comprensión Lectora

\begin{tabular}{|c|c|c|c|c|c|}
\hline \multicolumn{2}{|l|}{ Semestre } & Frecuencia & Porcentaje & $\begin{array}{l}\text { Porcentaje } \\
\text { válido }\end{array}$ & $\begin{array}{l}\text { Porcentaje } \\
\text { acumulado }\end{array}$ \\
\hline \multirow{4}{*}{ Primero } & Débil & 26 & 32,1 & 32,1 & 32,1 \\
\hline & Intermedio & 44 & 54,3 & 54,3 & 86,4 \\
\hline & Alto & 11 & 13,6 & 13,6 & 100,0 \\
\hline & Total & 81 & 100,0 & 100,0 & \\
\hline
\end{tabular}


En la tabla 2 se presenta la al eje temático de comprensión lectora donde se refleja un porcentaje débil del 32,1\% que concierne a las falencias del uso de las técnicas indicadas para una correcta comprensión lectora que se lleva a cabo en la redacción en los trabajos académicos. La comprensión, es el proceso que consiente en adquirir información, experimentar y estar consciente, lo cual admite la comunicación y también el éxito académico. (Silvia Trujillo, 2014)

Por otro lado, está el 54,3\% del ítem intermedio, demuestra que los estudiantes a menudo utilizan las técnicas adecuadas hacia una redacción en un trabajo académico, de manera que, conlleva un vocabulario técnico evitando así las muletillas, claramente con palabras apropiadas como también las clases de redacción para obtener escritos claros, precisos y elegantes, y por si fuese poco, investigar términos o palabras desconocidas para mayor entendimiento. El vocabulario es decisivo para la adquisición del lenguaje y la lectura, en particular, que cuando los estudiantes desconocen numerosas palabras, se incrementan las dificultades al procesar un texto. (Figueroa Sepúlveda \& Gallego Ortega , 2021) En el ítem alto con un bajo porcentaje de $13,6 \%$ en el cual demuestra que son pocos los estudiantes de primer semestre que abarcan una hábil comprensión lectora, el cual permite luego llegar a una correcta redacción en los trabajos académicos manteniendo las técnicas necesarias para el mismo, de modo que, se esmeran por hacerlo de la mejor manera posible con relecturas y repasos, siendo el resultado final satisfactorio ante el nivel académico. Por tanto, Gómez (2011) afirma que, la comprensión de textos está presente en todos los escenarios de los niveles educativos, por lo que se considera una actividad crucial para el aprendizaje; todo lo que los estudiantes adquieren y utilizan en los trabajos es a partir de los textos comprendidos.

Tabla 3 Normas APA

\begin{tabular}{|c|c|c|c|c|c|}
\hline \multicolumn{2}{|c|}{ Semestre } & Frecuencia & Porcentaje & $\begin{array}{c}\text { Porcentaje } \\
\text { válido }\end{array}$ & $\begin{array}{c}\text { Porcentaje } \\
\text { acumulado }\end{array}$ \\
\hline \multirow{3}{*}{ Primero } & Débil & 31 & 38,3 & 38,3 & 38,3 \\
& Intermedio & 45 & 55,6 & 55,6 & 93,8 \\
& Alto & 5 & 6,2 & 6,2 & 100,0 \\
\cline { 2 - 6 } & Total & 81 & 100,0 & 100,0 & \\
\hline
\end{tabular}

De la tabla 3 de la al eje temático de las normas APA, se puede constatar un puntaje medio de $38,3 \%$ en el ítem débil, por tanto, es un bajo conocimiento de las normas, dando a entender que existe un déficit al momento de aplicarlas en sus documentos académicos. 
"Estas normas permiten un estilo uniforme a los documentos escritos, evitan el plagio, permiten hacerles seguimiento, verificación y ampliación a las investigaciones, reconocer los créditos a los autores, igualmente ayudan a darle sentido, orden y fundamento" (Manual APA, 2016, citado en Gavalo y José, 2018, p. 20). Además, de dar solides y criterios a sus trabajos académicos.

El 55,6\%, del ítem intermedio se puede decir que, los estudiantes tienen un conocimiento básico, ya que, el "citar tiene una importancia fundamental y no simplemente porque consista en dar crédito a ideas de otras personas. La citación pone de manifiesto el ejercicio dialógico de los saberes" (Grupo Compás, 2020, p. 7). Es por ello que, desde los primeros semestres se debe enseñar con mayor énfasis la correcta aplicación de APA.

En el ítem que corresponde a alto, se presenta el más bajo puntaje, que es de 6,2\%, donde son pocos los estudiantes que aplican y muestran interés en aplicarlas de manera correcta, ya que, "pueden beneficiarse de dominar la escritura académica como una forma de desarrollar sus habilidades de pensamiento crítico y perfeccionar la precisión y claridad de su comunicación" (NORMASAPA.PRO, 2020, p. 7), permitiendo tener una comunicación efectiva en el aspecto académico, que le ayudará a mejorar esta habilidad, al momento de emplearlas en todos sus trabajos.

\section{CONCLUSIÓN O CONSIDERACIONES FINALES}

El estudio consistió en diagnosticar el nivel de redacción académica en los estudiantes del Primer semestre de la Carrera de Educación Inicial. Para ello, se realizó un estado del arte que facilitó definir el proceso de la redacción académica:

La redacción se ha vuelto fundamental para la realización de escritos, ya que, está compuesta por un conjunto de procesos que parten de elaboración, revisión y expresión de ideas, a su vez, demanda esfuerzo, así como, exactitud en la práctica. Por medio de ese proceso, los estudiantes construirán su conocimiento, involucrando el desarrollo del pensamiento crítico que les permitirá sintetizar, ordenar u organizar ideas para formar nuevos conceptos.

Los resultados evidencian que, el 84,6\% de estudiantes encuestados presentan dificultad en la aplicación de las normas de Ortografía lo que pone de manifiesto que aplican el uso ocasional de los signos de puntuación, acentuación, abreviaturas; lo cual, puede ser un impedimento para determinar cuándo y cómo aplicar cada una de estas normas, mismo 
que, origina errores en la escritura y, sobre todo, puede generar inconvenientes al redactar un escrito.

Por otro lado, se evidencia una dificultad en la Comprensión lectora por parte del discente, ya que, demuestra que tiene un nivel medio de análisis, síntesis, concentración e interés al interpretar el texto afectando su comunicación escrita. De igual manera, con respecto a las normas APA existen errores al momento de citar a los autores y de aplicar correctamente el formato sugerido, lo que lleva a una mala presentación de los escritos. Es importante que, las Instituciones de Educación Superior fortalezcan en los procesos pedagógicos las habilidades de redacción académica de los estudiantes, de tal forma que, elaboren trabajos de calidad, que contribuyan a sus aprendizajes y permitan el desarrollo óptimo de sus competencias profesionales.

\section{LISTA DE REFERENCIAS}

Araque Escalona, J., Viera Zambrano, Á., Corrales, N., \& Urrego, A. (2019). La Argumentación como Habilidad fundamental en el Proceso de Redacción Académica. Revista Cifra Nueva, 51-61.

Benítez Almirón, M. M. (2015). Influencia de las habilidades de escritura en la calidad de la redacción académica de los trabajos escritos de estudiantes de 3 ciclo, de la carrera de Ingienería electrónica en control y automatismo de la Universidad Católica de Santiago de Guayaquil. Tesis, Universidad Católica de Santiago de Guayaquil, Guayaquil. Obtenido de http://repositorio.ucsg.edu.ec/bitstream/3317/4249/1/T-UCSG-POS-MES-25.pdf

Cabanillas Huam, C. A. (Marzo de 2021). Estrategias y métodos para mejorar la ortografía. Polo del conocimiento , 6(3), 457 - 475. doi:10.23857/pc.v6i3.2381

Cabanillas-Huamán, C. A. (2021). Estrategias y métodos para mejorar la ortografía. Polo del conocimiento, 460 .

Castro Brown, Y., \& Pérez Padrón, M. C. (Junio de 2018). La construccion de textos escritos en la escuela cubana. Conrado, 7 -11. Obtenido de http://scielo.sld.cu/scielo.php?pid=S1990-

$86442018000200001 \&$ script=sci_arttext\&tlng=pt

Cerda Gutierrez, H. (1993). Los elementos de la investigación como reconocerlos, diseñarlos y construirlos. Quito: El buho ltda. 
Durán, R., Gómez, A., Sáchez, M., Aijón, M., Corrochano, D., Cruz, J., . . V Vicario, I. (2017). Guía didáctica para la elaboración de un trabajo académico. Iberoprinter. Obtenido de http://hdl.handle.net/10366/132754

Espinoza Centellas, F. (2017). Evaluación de la Calidad de la Redacción de los Estudiantes Cursantes del Primer Año Universitario en la UMSA. Edu. Sup. Rev. Cient. Cepies, 80-87.

Figueroa Sepúlveda, S., \& Gallego Ortega , J. (2021). Relación entre vocabulario y comprensión lectora: Un estudio transversal en educación básica. Revista Signos. Estudio e linguística , 54 (106).

Gabarró, D. (2011). Dominar la ortografía, . Barcelona, España: Academia Acelerating the world research. Obtenido de https://d1wqtxts1 xzle7.cloudfront.net/38347435/dominar-la-ortografia-LIBROTEORICO-with-cover-pagev2.pdf?Expires $=1639027196 \&$ Signature $=$ eBkDt7EwJ40CospBKP15KOHHqbc P52LV2dt8jngvYApVZOYgDffun8viNLKzFFkT4XeWg9hcFSzJ oZ6gxhXiST ov1nZJGUqMfrXdfnniDlGfSpBMoCtUKP7

Galicia Alarcón , L., Balderrama Trápaga, J., \& Edel Navarro, R. (2017). Validez de contenido por juicio de expertos: propuesta de una herramienta virtual. Apertura, 9(2), 42-45. Obtenido de http://www.scielo.org.mx/pdf/apertura/v9n2/20071094-apertura-9-02-00042.pdf

Gallego Valarezo, M. I. (2017). Normas APA como competencia de formación del Docente de Educación Básica en la elaboración de trabajos investigativos. Universidad técnica de Machala, Machala.

Gavalo, A., \& José, O. (2018). UnadApa, guía para facilitar el uso de las normas APA En los estudiantes de la UNAD.

Gómez Palomino, J. (2011). Comprensión lectora y rendimiento escolar: una ruta para mejorar la comunicación. Revista de investigación en comunicación y desarrollo, 27-36.

González. (2012). La ortografía en el aula . Káñina. Revista de Artes y Letras, 186.

González Hernández, K., Otero Paz, L., \& Castro Laguardia, A. M. (1 de Enero de 2016). Comprensión lectora, memoria de trabajo, fluidez y vocabulario en escolares 
cubanos. Actualidades Investigativas en Educación, 16(1), 1 - 18. doi:http://dx.doi.org/10.15517/aie.v16i1.21715

González Perón, A., Rodríguez Sánchez, M., \& Ledo Noguera, M. (2019). Enseñar a escribir en inglés: el enfoque proceso-producto orientado a la acción. Transformación, 15(1), $14 \quad$ - $\quad 26 . \quad$ Obtenido de http://scielo.sld.cu/scielo.php?script=sci_arttext\&pid=S207729552019000100014

Grupo Compás. (2020). Guía de citación y referenciación normas de redacción APA septima edición. Grupo Compás.

Gutiérrez -Rodríguez, M., \& Flórez -Romero, R. (2011). Enseñar a escribir en la universidad: saberes y prácticas de docentes y estudiantes universitarios. Revista Internacional de Investigación en Educación, 137-168.

Mantilla Falcón, L. M., \& Barrera Erreyes, H. M. (2021). La comprensión lectora. Un estudio puntual en la educación superior del Ecuador. Revista Latinoamericana

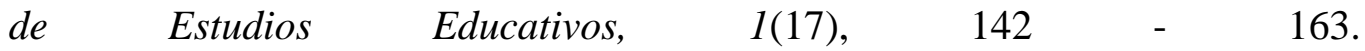
doi:https://doi.org/10.17151/rlee.2021.17.1.8

Manual de, E. (Enero de 2020). Normas de ortografía y redacción para publicaciones institucionales. Manual de Estilo. Obtenido de https://kitdigital.uc.cl/files/Manual_de_estilo_VRC_1pag.pdf

Monje, C. (2011). Metodología de la Investigación Cuantitativa y Cualitativa: Guía didáctica.

Montesdeoca Arteaga, D. V., Palacios Briones, F. E., Gómez Parra, M. E., \& Espejo Mohedano, R. (2021). Análisis de las habilidades ortográficas en español del estudiantado universitario en Ecuador. Revista Actualidades Investigativas en Educación, 21(1), 1 - 21. doi:10.15517/aie.v21i1.44074

Moreno, D., \& Carrillo, J. (2019). Normas APA 7. a edición Guía de citación y referenciación. Ediciones Universidad Central, 26.

NORMASAPA.PRO. (2020). Guía resumen del Manual de Publicaciones con Normas APA. NORMASAPA.PRO.

Ñañez, M., \& Lucas, G. (2017). Nivel de redacción de textos académicos de estudiantes ingresantes a la universidad. Opción: Revista de Ciencias Humanas y Sociales, 791-817. 
Orejudo González, J. P. (2019). Gamificar Tareas de Lectura en una Segunda Lengua: un Estudio Preliminar. Revista de Estudios y Experiencias en Educación, 99-100. doi:10.21703/rexe.20191836orejudo13

Romo M, P. E. (23 de diciembre de 2019). La comprensión y la competencia lectora. Universidad Central del Ecuador, 1(377).

Romo, P. (2019). La comprensión y la competencia lectora. Anales de la Universidad Central del Ecuador, 1(377), $165 . \quad$ Obtenido de https://revistadigital.uce.edu.ec/index.php/anales/article/view/2552/2859

Sampieri, R. H. (2014). Metodología de la investigación . México: McGRAW-HILL .

Sánchez Domínguez, J. P., \& Silva Zavala, E. M. (Julio de 2021). Caracterización de la Comprensión Lectora en estudiantes universitarios de nuevo ingreso. Dilemas $\begin{array}{llll}\text { contemp. } \quad \text { educ. } & \text { política }\end{array}$ doi:https://doi.org/10.46377/dilemas.v8i.2756

Silvia Trujillo, M. (enero-abril de 2014). Estudio de la comprensión lectora en latinoamérica: necesidad de un enfoque en la compresión. Innovación Educativa, 14(64).

Veliz Jara, N. C. (2020). Dificultades en la lectura y ortografía que afecta la escritura y el habla de los estudiantes del quinto año de Educacion General Básica de la Unidad Educativa Maria Andrea del cantón Babahoyo, Provincia de los Ríos. Trabajo especal de grado , Babahoyo. 